\title{
Investigation of swelling behaviors of GMZ bentonite pellet mixtures
}

\author{
Z. Zhang ${ }^{\text {i) }}$,W.M. Ye ${ }^{\text {ii) }}$, Z.R. Liu ${ }^{\text {i) }}$ \\ i) Department of Geotechnical Engineering, College of civil engineering, Tongii University, Shanghai, China \\ ii) Key Laboratory of Geotechnical and Underground Engineering of Ministry of Education, Tongji University, Shanghai, China
}

\begin{abstract}
Bentonite pellet mixtures have been recognized as an effective backfilling material for the sealing of boreholes and tunnels during the construction of engineering barrier system in deep geological disposal of high-level radioactive waste. Once installed in the repository, the complicated hydro-mechanical coupling conditions may affect the swelling properties of pellet mixtures. In this study, a series of swelling pressure tests and swelling deformation tests were conducted on GMZ bentonite pellet mixtures. Results showed a good linear relationship between final swelling pressure and void ratio in swelling pressure test. Similar phenomenon was also observed between the upper loading and void ratio in swelling deformation test. Furthermore, suction effects on swelling pressure and swelling strain of bentonite pellet mixture indicated that, at high suction stage (>4.2 MPa), the swelling of specimens was mainly controlled by the crystalline swelling and the exfoliation of clay particles. At lower suction stage $(<4.2 \mathrm{MPa})$, significant increases on swelling pressure and swelling strain were found, which might be related to osmotic swelling.
\end{abstract}

Keywords: high-level radioactive waste, deep geological disposal, bentonite pellet mixtures, swelling pressure, swelling deformation

\section{INTRODUCTION}

Bentonite pellet mixtures have been proposed as one of the candidate filling materials of tunnels and boreholes in geological disposal of high-level radioactive waste (HLW), not only because of its low permeability, high swelling capacity and high radionuclide adsorption properties, but also its easier backfilling operation, less compaction effort needed and more economic-efficiency expected (Imber and Villar et al., 2006; Alonso et al., 2011;Molinero-Guerra et al., 2016). Once installed in the repository, bentonite pellets will be submitted to coupled hydro-mechanical loadings. Bentonite pellets will absorb water and swell to fill some spaces existed in the repository. Then, the swelling of pellet mixture is restrained by host rocks and swelling pressure develops. Therefore, understanding of swelling behaviors of this type of material is important for assessing the overall function of the engineering barrier system.

The swelling behaviors of compacted pure bentonite powder or compacted bentonite/sand mixtures have been widely investigated. However, limited studies have been carried out on the swelling behavior of bentonite pellet mixtures. Imber \& Villar (2006) conducted swelling pressure tests on FoCa-clay pellet/powder (50/50) mixtures and concluded that the evolution of swelling pressure with time showed "double-peak" shaped phenomenon. Alonso et al. (2010) performed swelling pressure tests of FEBEX bentonite pellet mixtures by vapour equilibrium technique and concluded that the development curves of swelling pressure were mainly controlled by two concurrent effects, including the expansion of the pellets and the evolution of inter-pellet forces upon wetting. In addition, Hoffmann et al. (2004) conducted swelling deformation tests under constant-loading conditions and found that the initial collapse of immediately occurred after inundation, followed by an immediate period where the swelling strain continuously increased, and an additional compression deformation was observed when approaching to the saturation. Studies revealed that swelling behaviors of bentonite pellet mixtures were directly related to their multi-porosity structure. Hoffmann et al. (2007) performed Mercury intrusion porosimetry (MIP) tests on FEBEX bentonite pellet mixtures with different dry densities and three groups of pores could be identified, including intra-aggregate pores,inter-aggregate pores and inter-pellet pores. Results showed that compaction mainly affected the pore structure of inter-pellet pores. The visualization of hydration of FoCa-clay pellet/powder (50/50) mixtures under constant-volume conditions by microfocus X-ray computed tomography showed that the expansion of pellets upon hydration decreased the dry density of 
pellets and increased that of surrounding bentonite powder (Geet et al., 2005). By MIP tests, Alonso et al. (2011) observed that the pore structure of pellet mixtures evolved from an initial bimodal structure to a tri-modal structure with suction decreasing, involving complex volume change interactions among several groups of pores.

In China, Gao-Miao-Zi (GMZ) bentonite is currently considered as the first choice for using as buffer and backfill materials in high-level nuclear waste disposal program (Ye et al., 2009). Highly compacted GMZ bentonite pellets $\left(1.93 \mathrm{Mg} / \mathrm{m}^{3}\right)$ have been used to fill the gaps surrounding compacted bentonite blocks and studies showed that pellets not only influenced the variations of stress and relative humidity during hydration, but also might bring difficulties to the definition of boundary condition in numerical studies (Chen et al., 2014).

In this study, the main objective is to get insight into the swelling properties of GMZ bentonite pellet mixtures. Swelling pressure and swelling deformation tests were respectively conducted using different hydrating techniques and some interesting results were obtained.

\section{MATERIALS}

\subsection{GMZ bentonite}

GMZ bentonite tested in this study is extracted from Inner Mongolia of China, $300 \mathrm{~km}$ northwest from Beijing. X-ray diffraction test shows that GMZ Bentonite has montmorillonite content higher than $75.4 \%$. The liquid limit of GMZ bentonite is $313 \%$, the plastic limit is $38 \%$ and the specific gravity is 2.66 . the major exchangeable cations are $\mathrm{Na}^{+}$(29.66-38.48 $\mathrm{mmol} / 100 \mathrm{~g}), \mathrm{Ca}^{2+}(19.73-23.18 \mathrm{mmol} / 100 \mathrm{~g}), \mathrm{Mg}^{2+}$ (8.74-13.40 mmol/100 g) and $\mathrm{K}^{+}(0.47-1.01 \mathrm{mmol} / 100$ $\mathrm{g})$ and the total specific surface area is $570 \mathrm{~m}^{2} / \mathrm{g}$ (Ye et al., 2013).

\subsection{Bentonite pellet fabrication}

GMZ bentonite powder was suction controlled to $113 \mathrm{MPa}$ corresponding to water content $10.60 \%$ using vapor equilibrium technique. Then, bentonite powder was statically compacted into a specimen at a constant rate of $0.3 \mathrm{~mm} / \mathrm{min}$. After that, compacted bentonite was crushed into different groups and was controlled to a total suction of $113 \mathrm{MPa}$. The pore size distribution of a single bentonite is shown in Fig. 1. Atypical unimodal porosity distribution is observed, with a characteristic pore size of $19 \mathrm{~nm}$.

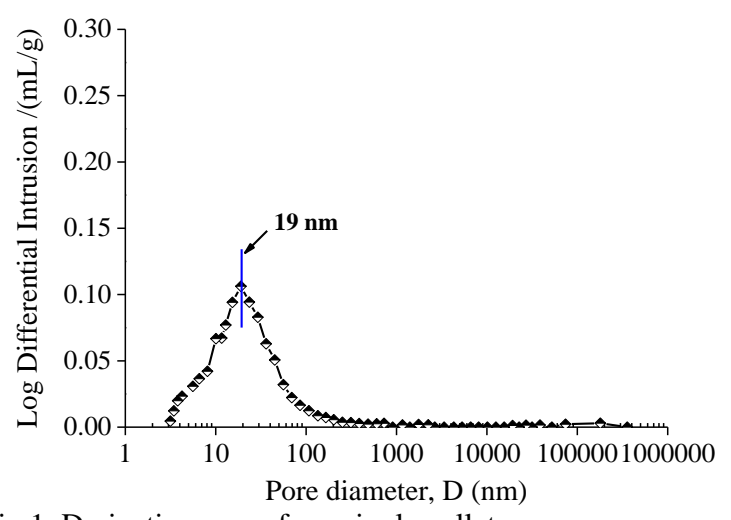

Fig.1. Derivative curve for a single pellet

\subsection{Sample preparation}

The grain size distribution curve of bentonite pellet mixture determined by Eq. (1) is presented in Fig.2.

$$
\mathrm{P}_{\mathrm{t}}=\left(\mathrm{d} / \mathrm{d}_{\max }\right)^{\mathrm{q}}
$$

where, $\mathrm{P}_{\mathrm{t}}$ is the fraction of the total solid with a particle size smaller than $d ; d_{\max }$ is the maximum particle size in the mixture; $\mathrm{q}$ is the distribution modulus with a value between 0 and 1. For the GMZ bentonite pellet mixtures tested in this study, $q=0.4$ and $\mathrm{d}_{\max }=7 \mathrm{~mm}$ could be adopted, respectively (Zhang et al., 2018).

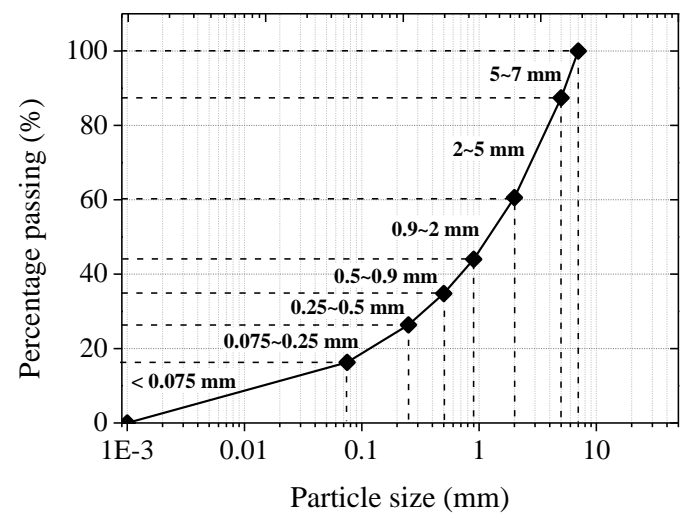

Fig.2. Particle size distribution curve of the pellet mixture

In this study, the sample size of $50 \mathrm{~mm}$ in diameter and $35 \mathrm{~mm}$ in height was used, and the pellet masses in different groups could be obtained for a given dry density of sample. Firstly, 5-7 $\mathrm{mm}$ and 2-5 $\mathrm{mm}$ pellets were weighed and mixed to obtain the coarse pellet part (> $2 \mathrm{~mm}$ ). In the same way, the rest groups were also respectively weighed and mixed to obtain the fine pellet part $(<2 \mathrm{~mm})$. Successively, the specimen was manufactured by alternating coarse pellet part and fine pellet part in three layers. After that, the specimen was vibrated for 3 mins on the vibrating table. Finally, the sample was compacted statically to achieve the target dry density.

\section{METHODS}

\subsection{Swelling pressure test}

The experimental setup employed in swelling 
pressure test includes three parts: (1) a stainless steel cell; (2) a data logging system; (3) a hydration system. The inner space of the cell is $50 \mathrm{~mm}$ in diameter and 60 $\mathrm{mm}$ in height for holding the specimen sandwiched between two porous stones. The data logging system is used to record the swelling pressure and the hydration system is employed for the infiltration of samples. In addition, for controlling the suction of samples, vapour equilibrium technique was employed.

\subsection{Swelling deformation test}

The oedometer cell used in swelling deformation tests has a size of $50 \mathrm{~mm}$ in diameter and $60 \mathrm{~mm}$ in height. A loading piston is employed to apply the vertical stress on the samples. A strain gauge with a precision of $1 \mu \mathrm{m}$ is fixed on the loading piston for monitoring the vertical deformation. Additionally, vapour equilibrium technique was used to control the suction of specimens. To apply zero suction, deionized water was circulated under the bottom of the specimens.

The experimental programme of swelling pressure and swelling deformation tests is listed in Table 1.

Table 1 . Test conditions

\begin{tabular}{cccc}
\hline Sample & $\begin{array}{c}\text { Initial dry } \\
\text { density } \\
\left(\mathrm{Mg} / \mathrm{m}^{3}\right)\end{array}$ & $\begin{array}{c}\text { Vertical } \\
\text { pressure } \\
(\mathrm{MPa})\end{array}$ & $\begin{array}{c}\text { Wetting } \\
\text { condition }\end{array}$ \\
\hline SP-01 & 1.25 & - & WC \\
SP-02 & 1.40 & - & WC \\
SP-03 & 1.45 & - & WC \\
SP-04 & 1.60 & - & WC \\
SP-05 & 1.70 & - & WC \\
SP-06 & 1.45 & - & VET \\
SD-01 & 1.45 & 0.1 & WC \\
SD-02 & 1.45 & 0.4 & WC \\
SD-03 & 1.45 & 0.8 & WC \\
SD-04 & 1.45 & 1.94 & WC \\
SD-05 & 1.45 & 0.4 & VET \\
SD-06 & 1.45 & 0.8 & VET \\
\hline SP
\end{tabular}

$\mathrm{SP}=$ swelling pressure, $\mathrm{SD}=$ swelling deformation, $\mathrm{VET}=$ vapour equilibrium technique, $\mathrm{WC}=$ water circulation.

\section{RESULTS AND DISCUSSION}

\subsection{Swelling pressure}

\subsubsection{Dry density effect}

Evolutions of swelling pressure with time for SP-01, SP-02, SP-03, SP-04 and SP-05 are shown in Fig.3. It is observed that the swelling pressure of low dry density specimens shows a continuous increase on swelling pressure. While for high dry density specimens (SP-04 and SP-05), a double-peak characteristic was observed. Swelling pressure increased rapidly at the initial hydration stage, followed by an immediate period where swelling pressure decreased, and then swelling pressure increased again with a lower rate and reached the equilibrium state when approaching to saturation state. In addition, a good linear relationship between final swelling pressures and void ratio is shown in Fig.4.

For low dry density specimens, the arrangement of pellets was loose and the expansion was mainly contributed to filling surrounding voids of pellets, resulting in low inter-pellet force. Under low swelling pressure, the expansion of pellets upon hydration prevails over the collapse of overall structure and a continuous increase on swelling pressure was observed. With the increase of dry density, the compacted loading of specimen increased and the arrangement of pellets became tighter. The initial wetting resulted in higher increase on swelling pressure. As wetting processed, the granular structure of pellet mixture underwent a collapse under high swelling pressure (the first peak) and swelling pressure decreased. After that, the further hydration led to the second increase of swelling pressure. This phenomenon might be explained as that the swelling of pellets prevailed over the collapse of the overall structure. The stable state on swelling pressure represented the equilibrium between the expansion of pellets and the collapse of granular structure.

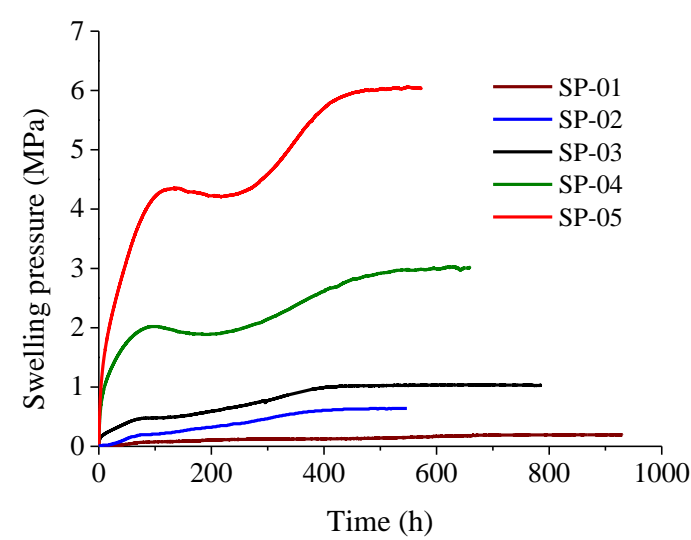

Fig. 3. Evolutions of swelling pressure for specimens with different dry densities

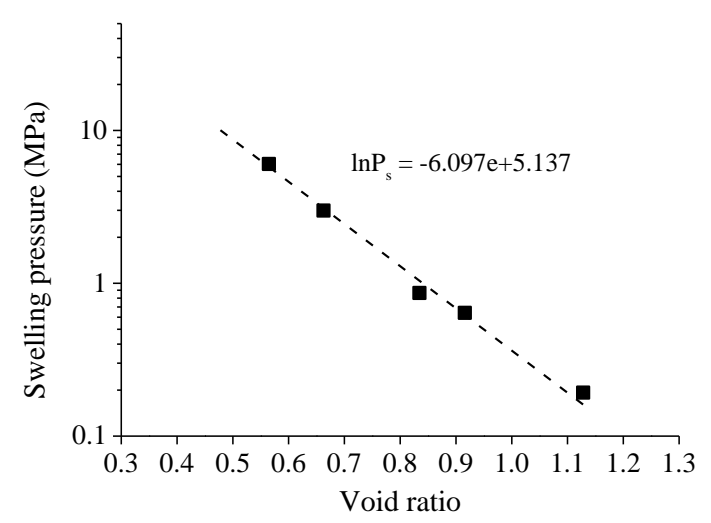

Fig. 4. Evolutions of final swelling pressure with void ratio

\subsubsection{Suction effect}


The change of swelling pressure with time for SP-06 is presented in Fig.5. A rapid increase of swelling pressure was observed for suction decreasing from the initial value to $38 \mathrm{MPa}$. With the further reduction on suction $(38 \mathrm{MPa} \rightarrow 9 \mathrm{MPa} \rightarrow 4.2 \mathrm{MPa})$, a slight increase on swelling pressure was obtained. Once being wetted by the infiltration of distilled water, swelling pressure rapidly increased and reached the final value of 0.97 MPa.

The evolution of swelling pressure with suction can be interpreted by the hydration mechanism of smectites. At high suction stage, the expansion of pellets mainly contributed to the crystalline swelling and the stiffness of pellet was high. Consequently, an apparent increase on swelling pressure was observed. With the decrease of suction, the exfoliation of clay particles occurred and macro-pores were significantly filled by the expansion of pellets (Saiyouri et al., 2000; Ye et al., 2009). During this stage, the structure of pellet mixture underwent the collapse, thus a slight increase on swelling pressure was observed. When the mixture was hydrated by injecting distilled water, the suction decreased rapidly and the swelling pressure of specimens was controlled by the osmotic swelling. Therefore, a significant increase was observed.

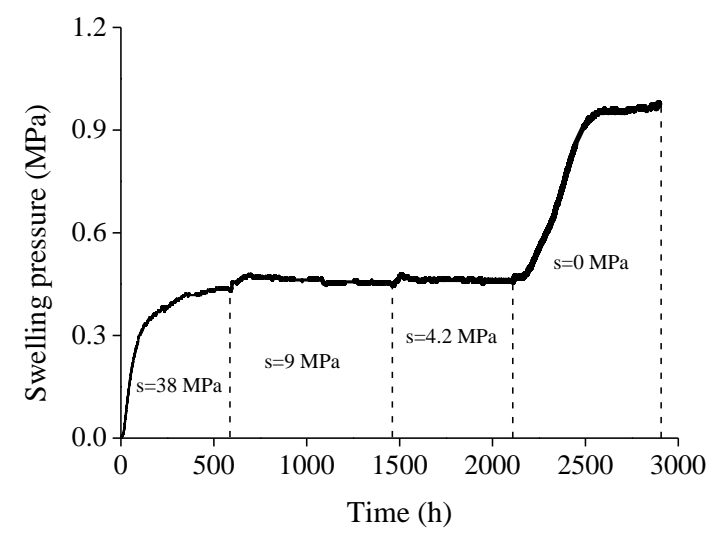

Fig. 5. Evolutions of swelling pressure with time for SP-06

\subsection{Swelling deformation}

\subsubsection{Upper loading effect}

Evolutions of swelling strain with time for specimens SD-01, SD-02, SD-03 and SD-04 are shown in Fig.6. Under low upper loading conditions, swelling strain increased quickly at the initial stage and then gradually stabilized. But at high loading condition (SD-04), the initial hydration resulted in a compression of specimen. As the wetting processed, a slight swelling strain was observed. In addition, similar to swelling pressure test results, a good linear relationship between the final swelling strain and upper loadings is found in Fig.7. The void ratio at the initial state corresponded to the upper loading $0.798 \mathrm{MPa}$, lower than swelling pressure obtained under constant-volume conditions.

Due to low upper loading, the expansion of pellets upon hydration was more significant, resulting in a higher swelling rate and a bigger swelling strain. But under a high upper loading, the granular structure might undergo a collapse during the initial hydration stage. The further hydration resulted in the swelling of specimen. This could be explained as that the expansion of pellets prevailed over the collapse of granular structure. Furthermore, swelling pressure of specimen obtained from swelling deformation test was lower than swelling pressure obtained under constant-volume condition, which might be related to different hydration paths.

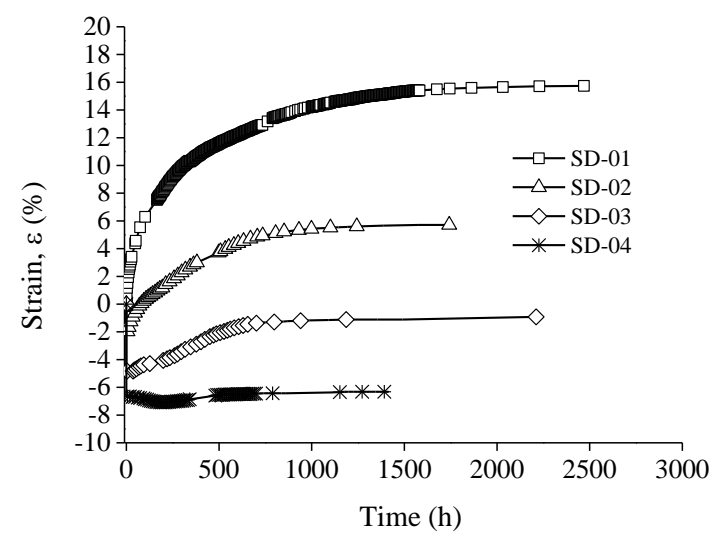

Fig. 6. Evolutions of swelling strain with time at different upper loadings conditions

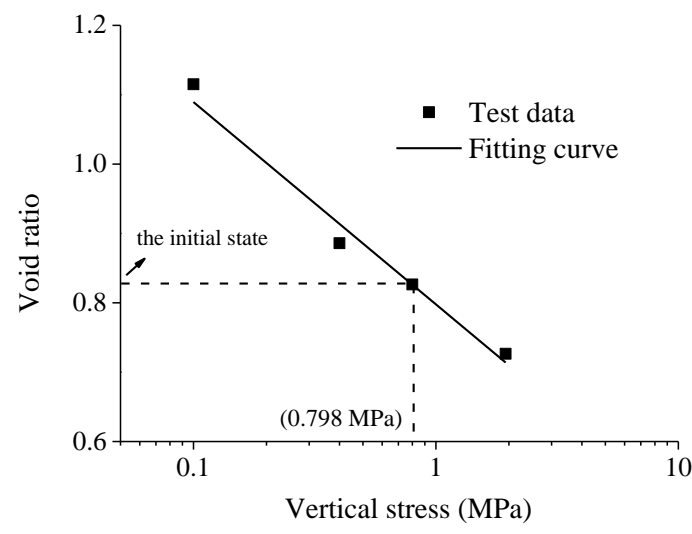

Fig. 7. Evolutions of final swelling strain with void ratio

\subsubsection{Suction effect}

Evolutions of swelling strain with time for SD-05 and SD-06 are presented in Fig.8. As suction decreased from the initial state to $4.2 \mathrm{MPa}$, void ratio of specimen SD-05 only increased from 0.807 to 0.833 . However, when the specimen was wetted to the saturation state by injecting distilled water, a significant increase on void ratio was observed and eventually reached its final value 0.905 . For SD-06, as suction decreased to 38 $\mathrm{MPa}$, a collapse was observed with void ratio correspondingly decreased from 0.773 to 0.729 . Then, with the further decrease of suction, void ratio continuously increased and eventually reached a void ratio 0.810 at the saturation state. 
Based on the swelling mechanism of compacted smectites, the change of swelling strain at high suction stage (> $4.2 \mathrm{MPa}$ ) was mainly controlled by the crystalline swelling and the exfoliation of clay particles. A continuous increase on swelling strain of SD-05 indicated that the expansion of pellets prevailed over the collapse of overall structure resulted from the exfoliation of clay particles. But for SD-06, an apparent compression occurred, which might be contributed to the rearrangement and the crushing of pellets. As suction decreased to lower value $(<4.2 \mathrm{MPa})$, curves showed a significant swelling of samples. This could be related to the osmotic swelling.

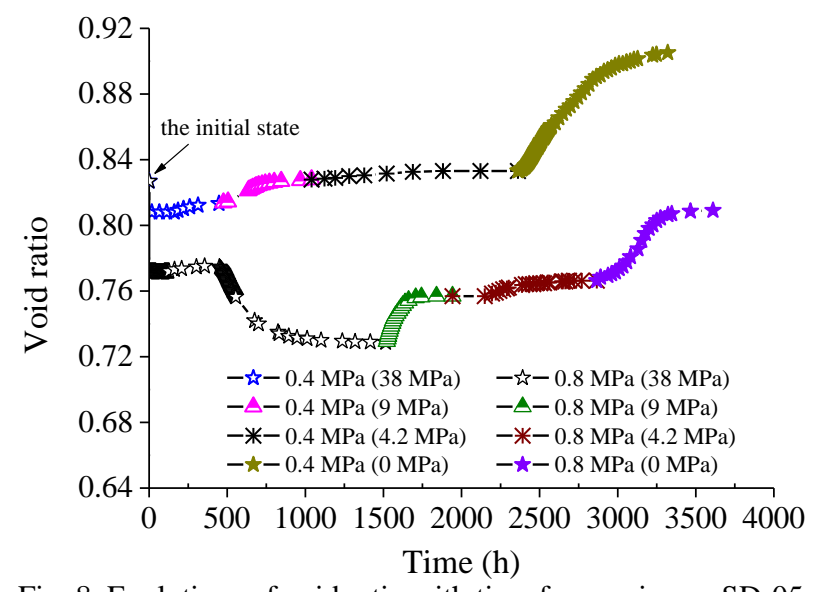

Fig. 8. Evolutions of void ratio with time for specimens SD-05 and SD-06

\section{CONCLUSION}

The swelling properties of GMZ bentonite pellet mixtures were investigated by performing swelling pressure test and swelling deformation tests. Dry density effect and suction effect on swelling pressure and upper loading effect and suction effect on swelling deformation were respectively analyzed and discussed. A good linear relationship was obtained between swelling pressure and void ratio. Similarly, a good linear relationship was also observed between swelling strain and upper loading. At high suction stage, the swelling property of pellet mixture was mainly controlled by the crystalline swelling and the exfoliation of clay particles. Consequently, low swelling pressure and swelling strain was observed. When suction decreased to lower value, significant swelling occurred, which might be related to osmotic swelling.

\section{ACKNOWLEDGEMENTS}

The authors are grateful to the National Natural Science Foundation of China (41527801, 41672271) for the financial supports.

\section{REFERENCES}

1) Alonso, E.E., Hoffmann, C. and Romero, E. (2010): Pellet mixtures in isolation barriers, Journal of Rock Mechanics \& Geotechnical Engineering, 2(1), 12-31.

2) Alonso, E. E., Romero, E. and Hoffmann, C. (2011): Hydromechanical behaviour of compacted granular expansive mixtures: experimental and constitutive study, Géotechnique, 61(4), 329-344.

3) Chen, L., Liu, Y.M., Wang, J., Cao, S.F., Xie, J.L. and Ma, L.K. (2014):Investigation of the thermal-hydro-mechanical (thm) behavior of gmz bentonite in the china-mock-up test, Engineering Geology, 172, 57-68.

4) Geet, M.V., Volckaert, G. and Roels, S. (2005): The use of microfocus $\mathrm{x}$-ray computed tomography in characterising the hydration of a clay pellet/powder mixture, Applied Clay Science, 29(2), 0-87.

5) Imbert, C. and Villar, M.V. (2006): Hydro-mechanical response of a bentonite pellets/powder mixture upon infiltration, Applied Clay Science, 32(3-4), 197-209.

6) Hoffmann, C., Alonso, E.E. and Romero, E. (2004), Fabric changes of a pellet-based bentonite buffer material and its effect on mechanical behavior, Elsevier Geo-Engineering Book Series, 2(147), 341-346.

7) Hoffmann, C., Alonso, E.E. and Romero, E. (2007): Hydro-mechanical behaviour of bentonite pellet mixtures, Physics \& Chemistry of the Earth Parts $\mathrm{A} / \mathrm{b} / \mathrm{c}, 32(8)$, 832-849.

8) Molinero-Guerra, A., Mokni, N., Delage, P., Cui, Y.J., Tang, A.M., Aimedieu, P., Bernier, F. and Bornert, M. (2016): In-depth characterisation of a mixture composed of powder/pellets MX80 bentonite, Applied Clay Science, 135.

9) Saiyouri, N., Hicher, P.Y. and Tessier, D. (2000): Microstructural approach and transfer water modelling in highly compacted unsaturated swelling clays, Mechanics of Cohesive - frictional Materials, 5(1), 42-60.

10) Zhang, Z., Ye, W.M., Liu Z.R., Chen, B. and Cui Y.J. (2018): Influences of PSD curve and vibration on the packing dry density of crushed bentonite pellet mixtures, Construction and Building Materials, 185:246-255.

11) Ye, W.M., Cui, Y.J., Qian, L.X. and Chen, B. (2009): An experimental study of the water transfer through confined compacted GMZ bentonite, Engineering Geology, 108(3), 169-176.

12) Ye, W.M., Zhang, Y.W., Chen, Y.G., Chen, B. and Cui, Y.J. (2013): Experimental investigation on the thermal volumetric behavior of highly compacted gmz01 bent, Applied Clay Science, 83-84, 210-216. 